\title{
Computed tomography of the brain, chest, and abdomen in the preoperative assessment of non-small cell luing cancer
}

\author{
D GRANT, D EDWARDS, P GOLDSTRAW \\ From University College Hospital and Brompton Hospital, London
}

\begin{abstract}
The benefit to be gained from carrying out computed tomography of brain and abdomen in addition to the chest has been evaluated retrospectively in 114 consecutive patients with non-small cell lung cancer who, on the basis of history, clinical examination, chest radiography, and bronchoscopy had been considered potentially operable. Computed tomography of the chest showed potentially inoperable tumour in 37 patients, of whom 25 had tumour confined to the chest. Three patients were shown to have malignant disease within the mediastinum and abdomen; five within the mediastinum and brain; and four within the mediastinum, abdomen, and brain. Computed tomography of the abdomen disclosed deposits in nine patients, but in only two were the abnormalities restricted to the abdomen. Computed tomography of the brain showed metastases in 10 patients, of whom only one had metastatic disease confined to the brain. Thus three patients had isolated deposits in the abdomen and brain. In 12 patients the identification of metastases in the abdomen and brain removed the need for mediastinoscopy. Preoperative computed tomography of the abdomen and brain detected occult metastases in 15 patients $(13 \%)$ in this study. In three patients the extrathoracic abnormality proved the only contraindication to surgery, but in the other 12 it provided valuable corroborative evidence of incurability and facilitated the assessment of the mediastinal abnormality.
\end{abstract}

\section{Introduction}

The aim of surgery in lung cancer is to effect cure or to extend survival far beyond that which may be achieved with other forms of treatment. Incomplete or "palliative" resection confers no survival advantage ${ }^{1}$ and complete resection should be the aim for all patients. Preoperative evaluation attempts to identify and exclude from surgery patients with mediastinal tumour or distant metastases. Extensive mediastinal disease produced by invasion or glandular metastases considerably reduces the prospect of complete resection and in these circumstances cure is improbable. ${ }^{23}$ Evaluation of the mediastinum by cervical mediastinoscopy supplemented by left anterior mediastinotomy for left upper lobe tumours has become established as an important step before thoracotomy. Com-

Address for reprint requests: Mr Peter Goldstraw, Thoracic Surgery, Brompton Hospital, London SW3 6HP.

Accepted 16 August 1988 puted tomography of the mediastinum has been extensively evaluated by us and others. ${ }^{45}$ Computed tomography will occasionally disclose mediastinal invasion so extensive as to satisfy the most sceptical, but in most cases radiological abnormalities suggesting invasion or lymphadenopathy require confirmation by surgical evaluation of the mediastinum. It is now accepted that when a computed tomography scan of the mediastinum is normal mediastinal exploration may be omitted before thoracotomy, ${ }^{4}$ and this alone justifies the routine use of computed tomography in preoperative evaluation. After "complete" resection most patients who relapse have tumour at a distant site, ${ }^{6}$ presumably as a result of occult metastases that have eluded preoperative recognition. The preoperative search for distant metastases requires a careful history to elicit recent bone pain or personality change, and clinical examination to find hepatomegaly, bone tenderness, or palpable cervical lymphadenopathy. Non-specific indicators that have been identified ${ }^{7}$ include weight loss (greater than $3 \mathbf{~ k g}$ in six months), unexplained anaemia (haemoglobin 
less than $11 \mathrm{~g} / \mathrm{dl}$ ), abnormal results in liver function tests, and hypercalcaemia. Where these non-specific features are present metastases are more likely and isotope scans of brain, liver, and bone have been shown to be of value. ${ }^{78}$ For the detection of metastases computed tomography has been shown to be the most sensitive technique for the liver, ${ }^{9}$ more accurate than isotope scans in the brain, ${ }^{10}$ and unrivalled for the adrenal glands or para-aortic nodes. ${ }^{11-13}$ We have routinely added computed tomography of the brain and abdomen to that of the chest in an attempt to identify metastases that would otherwise not have been detected preoperatively. We present a retrospective review of 114 consecutive patients considered suitable for surgical treatment with a known or presumed diagnosis of non-small cell lung cancer.

\section{Methods}

All patients with a known or presumed diagnosis of lung cancer were evaluated by history, clinical examination (including neurological examination), chest radiography, and bronchoscopy. Where non-specific features suggested occult metastases isotope scanning of the skeleton was undertaken. Patients otherwise considered operable were routinely referred for computed tomography of the brain, chest, and abdomen by the referring physician or thoracic surgeon. All scans were performed with a Philips Tomoscan 310 scanner with a $9 \mathrm{~mm}$ slice thickness and two to four second scan time. Scanning started at the lung apices and proceeded in one and a half slice increments to the level of the aortic arch above the pulmonary hila. At this point $16 \mathrm{~g}$ of iodine was rapidly injected into an antecubital vein. Contiguous and rapid sequence scanning was performed through the hilar regions, reverting to one and a half slice increments down to the lower pole of the kidneys. Nine millimetre contiguous cuts were then undertaken through the brain. All scans were reviewed by one radiologist (DE). Indeterminate or questionable reports were not allowed.

The following appearances on the computed tomography scan were potential contraindications to curative resection: (1) mediastinal lymph glands larger than $15 \mathrm{~mm}$ in either axis; (2) mediastinal invasion suggested by contiguity between tumour and mediastinal structures with loss of the normal tissue plane; (3) contralateral pulmonary deposits; (4) chest wall invasion medial to the posterior rib angle; (5) thoracic skeletal deposits; (6) extrathoracic metastases in the abdomen or brain.

Abnormalities on the computed tomography scan of the mediastinum were evaluated by surgical exploration, cervical mediastinoscopy being supplemented by left anterior mediastinotomy where the tumour lay in the left upper lobe. Isolated abnor- malities within the liver or adrenals were evaluated by fine needle aspiration biopsy under computedo tomography guidance when feasible.

\section{Results}

One hundred and fourteen consecutive patients $\overrightarrow{-}$ entered the study over 14 months. Tissue confirmationof malignancy was not always available at the time of $\vec{\omega}$ computed tomography but histological diagnoses? were subsequently obtained for all 114 patients on the $x$ basis of tissue specimens taken at bronchoscopy, $\vec{\omega}$ mediastinoscopy, or thoracotomy. Patients with smallcell lung cancer were excluded from the study. Histo-io logically squamous cell carcinoma was found in $77 \mathrm{\omega}$ patients, adenocarcinoma in 21 , and large cell un-응 differentiated carcinoma in 16. None of the patientshad clinical evidence to suggest metastases but non- $Z$ specific features were present in eight patients, all of whom had normal bone isotope scans.

Computed tomography scans of the mediastinum were considered abnormal in 37 patients (fig 1). Of these, 20 patients had squamous carcinoma, seven $\infty$ adenocarcinoma, and 10 undifferentiated large cell ${ }^{\circ}$ carcinoma. Fifteen of these 37 patients did not undergo further evaluation of the mediastinum, in three cases because they refused surgery and in $12 \frac{}{\circ}$ because abnormalities on the brain or abdomeno computed tomography scan suggested metastatic dis $-\frac{\mathbb{Q}}{\Omega}$ ease. Thus 22 of the 37 patients with an abnormal computed tomography scan of the mediastinum 3 proceeded to surgical evaluation (fig 2). Mediastinoscopy was undertaken in 21 patients. In 16 of these mediastinoscopy confirmed the computed tomo graphy abnormality and in 15 the disease was con-0응 sidered to be inoperable. Seven patients underwent thoracotomy. In one patient mediastinoscopy was noto

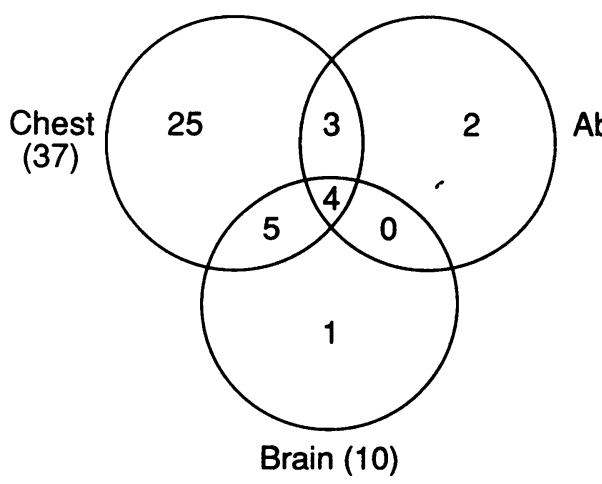

Total studied 114 patients

Fig 1 Computed tomography identification of nonresectable chest and metastatic disease by region. 


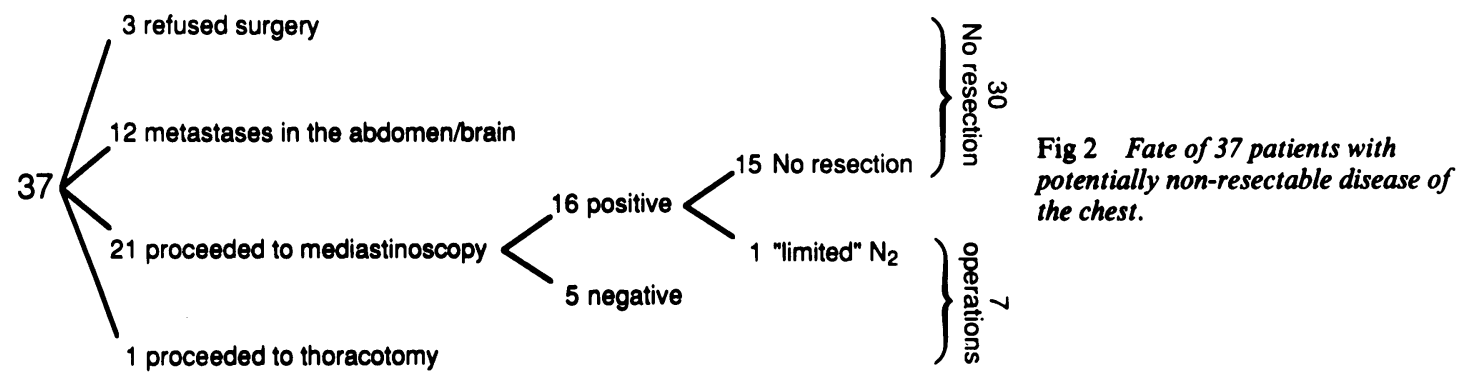

undertaken despite the computed tomography evidence of mediastinal node disease; thoracotomy confirmed that the glands contained tumour. In one patient the gland disease seen by mediastinoscopy was considered sufficiently localised not to preclude surgical resection. In the remaining five patients mediastinal exploration showed no malignancy and thoracotomy was undertaken. Resection was possible in all five patients, but limited mediastinal node disease was confirmed in two patients.

Computed tomography of the abdomen showed occult malignancy in nine patients (fig 1). Metastases were located within the adrenal gland in three, the kidney in one, the liver in one, and a para-aortic lymph node in one, and one was a lumbar vertebral deposit; two patients had deposits in both the adrenal gland and the liver. In seven of the nine patients computed tomography of the mediastinum suggested extensive disease. In two patients isolated abdominal metastases were investigated by fine needle aspiration biopsy, which confirmed an adrenal deposit in one patient and a secondary tumour in the liver in another. Resection was not undertaken in any of these nine patients.

Computed tomography of the brain showed metastases in 10 patients (fig 1 ). In nine of these patients, however, other contraindications to surgery were found on the computed tomography scan of the chest or abdomen, or both; in only one patient was the abnormality restricted to the brain. None of these patients underwent resection.

In 74 patients computed tomography of the brain, chest, and abdomen did not show any contraindication to curative resection. Mediastinal exploration was considered unnecessary in these patients and resection was undertaken in all 74.

\section{Discusedon}

The preoperative evaluation of patients with lung cancer attempts to identify those with mediastinal tumour or distant metastases in whom resection would be futile. Computed tomography scanning of the chest is of established value in the preoperative evaluation of the mediastinum. ${ }^{45}$ Variation in the computed tomography size criterion or the technique of evaluating mediastinal glands can alter the sensitivity of the investigation..$^{14}$ Reducing this size criterion to $1 \mathrm{~cm}$ or less will increase the sensitivity at the cost of decreasing the specificity of the observation. ${ }^{14}$ Both sensitivity and specificity may be increased if the radiologist is allowed to declare difficult scans to be "indeterminate"15 but such statistical manipulation is of little value to the clinician.

In our study the results of computed tomography of the chest potentially contraindicated resection in 37 of our 114 patients (32\%). Abnormalities were limited to the scan of the chest in 25 patients, of whom three refused further evaluation. Mediastinal exploration was performed in 21 patients. The remaining patient proceeded directly to surgery and the surgeon's decision to disregard the computed tomography appearances without undertaking mediastinal exploration was clearly erroneous. Mediastinal exploration confirmed the abnormality seen on the scan of the mediastinum in 16 patients and 15 of these did not proceed to thoracotomy. Resection was performed in the other patients and also in five patients in whom mediastinal exploration showed no malignancy. In three of the patients no mediastinal disease was seen at surgery and in the other three N2 disease was confirmed but the localised nature of this was not considered to preclude resection. ${ }^{16}$ In 12 patients mediastinal abnormalities on the computed tomography scan were not evaluated by mediastinal exploration as there was evidence of metastasis on the scan of the abdomen or brain or both. This may explain why the proportion of patients with abnormal computed tomography scans of mediastinum in this study who proceeded to resection is much lower than that reported in an early series from our group. ${ }^{4}$ In the present study 25 patients had computed tomography evidence of inoperability confined to the chest scan. Only 21 underwent mediastinal exploration, of whom only six proceeded to resection. In our earlier study 23 patients with abnormal scans of the mediastinum were subjected to mediastinal exploration, of whom nine underwent resection." We cannot be sure how many of the 12 patients with abnormal computed tomography scans 
of the abdomen or brain in addition to the mediastinum would have undergone mediastinal exploration and subsequent resection if only chest scans had been performed. Extending the preoperative computed tomography scan from the chest to include the abdomen and brain would appear at first sight to be of little value as only three patients had isolated extrathoracic metastases detected, in the abdomen in two cases and in the brain in one. In these three cases, however, the discovery of extrathoracic metastases undoubtedly saved futile surgery with its attendant risks. Whether the saving of unnecessary surgery in these three patients justifies extending preoperative computed tomography of the chest by $\mathbf{3 0}$ minutes to include the abdomen and brain is a matter of judgment. For us, however, the issue is resolved by considering the fate of the 12 patients who had malignant disease in the abdomen or brain or both in addition to the mediastinum. Three patients had computed tomography evidence of tumour in the mediastinum and abdomen; five in the mediastinum and brain; and four in the mediastinum, abdomen, and brain. If computed tomography of the brain and abdomen had not been carried out all 12 would have required inpatient evaluation and surgical assessment of the mediastinum and some would have undergone inappropriate surgical resection.

Thus in 114 consecutive patients with lung cancer routine computed tomography of the abdomen and brain before surgery showed that 15 patients $(13 \%)$ had extrathoracic deposits - abdominal metastases in five, cerebral metastases in six, and both cerebral and abdominal deposits in four patients, only three of the extrathoracic deposits being isolated metastases. Futile surgery was saved in these three patients, and in the other 12 surgical exploration of the mediastinum was considered unnecessary and in some of these futile surgery may have been prevented.

\section{References}

1 Rocmans P, Defrancquen P. Resultats du traîtement chirurgica: 1-Du cancer bronchique. Rev Med Brux 1981;2:107-13.

2 Gibbons JRP. The value of mediastinoscopy in assessing operability in carcinoma of the lung. $\mathrm{Br} J$ Dis Chest 1972;66:162-6.

3 Viikari SJ, Inberg MV, Puhakka H, Linna MI, Vanttinen
E. The role of mediastinoscopy in the treatment of lungs? carcinoma. Bull Soc Int Chir 1974;2:119-26.

4 Goldstraw P, Kurzer M, Edwards D. Preoperative stag ing of lung cancer: accuracy of computed tomography, versus mediastinoscopy. Thorax 1983;10:10-5.

5 Goldstraw P. CT scanning in the preoperative assessmen? of non-small cell lung cancer. In: Hansen HH, ed. Lungs cancer: basic and clinical aspects. Boston: Nijholl, $1986 \vec{\circ}$ 183-99.

6 Immerman SC, Vanecko RM, Fry WA, Head LR $\overrightarrow{\dot{c}}$ Shields TL. Site of recurrence in patients with stages $\mathrm{p}$ and 11 carcinoma of the lung resected for cure. Ann Thorac Surg 1981;32:23-7.

7 Hoper RG, Beechler CR, Johnson MC. Radioisotopes scanning in the initial staging of bronchogenic carci $-\overrightarrow{-}$ noma. Am Rev Respir Dis 1978;118:279-86.

8 Ramsdell JW, Peters RM, Taylor AT, Alazarki NP, Tist GM. Multiorgan scans for staging cancer: correlation with clinical evaluation. $J$ Thorac Cardiovasc Surg 1977;73:653-9.

9 Gunvein P, Makuuchi M, Takayasu K, Moriyama Nơ Yamasaki S, Hasagawa H. Preoperative imaging of liver metastases: comparison of angiography, CT scan and ultrasonography. Ann Surg 1985;202:573-9.

10 Lusins JO, Chayes Z, Nakagawa H. Computed tomogra phy and radionucleide brain scanning: comparison inoo evaluating metastatic lesions to the brain. NY State $\rho^{\circ}$ Med 1980;80:185-9.

11 Sandler MA, Pearlberg KL, Madrazo BL, Gitschlag KF Gross SC. Computed tomographic evaluation of the adrenal gland in the preoperative assessment of bron chogenic carcinoma. Radiology 1982;145:733-6.

12 Nielsen ME, Heaston DK, Dunnick NR, Korobkin M@ Preoperative CT evaluation of adrenal glands in non $\overrightarrow{\bar{\sigma}}$ small cell bronchogenic carcinoma. AJR 1982;139: 317-20.

13 Pagani JJ. Non-small cell carcinoma adrenal metastases? computed tomography scanning and percutanious needle biopsy in their diagnosis. Cancer 1984;53 $1058-60$.

14 Osborne DR, Korobkin M, Ravin CE, et al. Comparison of plain radiography, conventional tomography, and 3 computed tomography in detecting intrathoracic lymph node metastases from lung carcinoma. Radiology 1982;142:157-61.

15 Baron RL, Levitt RG, Sagel SS, White MJ, Roper CL음 Narbarger JP. Computed tomography in preoperative $D$ evaluation of bronchogenic carcinoma. Radiolog 1982;145:727-32.

16 Pearson FG, Delarue NC, Ilves R, Todd TRJ, Coopero JD. Significance of positive superior mediastinal nodes identified at mediastinoscopy in patients with resect able cancer of the lung. J Thorac Cardiovasc Surg 1982 W 83:1-11. 